\title{
Indirect and direct measurement of thermal neutron acceleration by inelastic scattering on the ${ }^{177} \mathrm{Lu}$ isomer
}

\author{
G. Bélier 1,a, O. Roig ${ }^{1}$, V. Méot ${ }^{1}$, J. Aupiais ${ }^{2}$, J.-M. Daugas ${ }^{1}$, Ch. Jutier ${ }^{2}$, G. Le Petit ${ }^{2}$, A. Letourneau ${ }^{3}$, \\ F. Marie ${ }^{3}$, and Ch. Veyssière ${ }^{4}$ \\ 1 CEA/DIF/DPTA Service de Physique Nucléaire, BP. 12, 91680 Bruyères-le-Châtel, France \\ ${ }^{2}$ CEA/DIF/DASE Service de Radio-analyse, Chimie et Environnement, BP. 12, 91680 Bruyères-le-Châtel, France \\ 3 CEA/DSM/DAPNIA Service de Physique Nucléaire, CE Saclay, 91191 Gif-sur-Yvette, France \\ ${ }^{4}$ CEA/DSM/DAPNIA Service d'Ingénierie des Systèmes, CE Saclay, 91191 Gif-sur-Yvette, France
}

\begin{abstract}
When neutrons interact with isomers these last can be de-excited. In such a reaction the outgoing neutron has an energy greater than the ingoing one. This process is referred as Inelastic Neutron Acceleration or Superelastic Scattering. Up to now this process was observed for only two nucleus, ${ }^{152 \mathrm{~m}} \mathrm{Eu}$ and ${ }^{180 \mathrm{~m}} \mathrm{Hf}$ by measuring the number of fast neutron produced by isomeric targets irradiated with thermal neutrons. In these works the energies of the accelerated neutrons were not measured. This report presents an indirect measurement of inelastic neutron acceleration on ${ }^{177 \mathrm{~m}} \mathrm{Lu}$, based on the burn-up and the radiative capture cross sections measurements. Since at thermal energies the inelastic scattering and the radiative capture are the only processes that contribute to the isomer burn-up, the inelastic cross section can be deduced from the difference between the two measured quantities. Applying this method for the ${ }^{177} \mathrm{Lu}$ isomer with different neutron fluxes we obtained a value of 258 (58) barns, and determined that there is no integral resonance for this process. In addition the radiative capture cross section on ${ }^{177 \mathrm{~g}} \mathrm{Lu}$ was measured with a much better accuracy than the accepted value. Since the acceleration cross section is quite high a direct measurement of this process was undertaken, sending thermal neutrons and measuring the fast neutrons. The main goal now is to measure the outgoing neutron energies in order to identify the neutron transitions in the exit channel. In particular the K conservation question can be addressed by such a measurement.
\end{abstract}

\section{Introduction}

Nuclear reactions involving isomers are relevant to many topics in nuclear physics [1] such as nuclear reaction mechanisms, nuclear structure, inelastic neutron scattering, astrophysics yields, and nuclear reactor development. When the isomer half-lives are long enough, target preparation is possible and different types of experiments can be performed, for example Coulomb excitation [2], photonuclear reaction $[3,4]$, transfer reactions $[5,6]$, or neutron capture. In particular many long-lived $\mathrm{K}$ isomers are known in the $\mathrm{A}=180$ mass region, and these isomers represent a unique chance for energy storage. Their radiative decay rates do not result only from the spin and energy difference with the lower states, but these transitions are hindered by the $K$ quantum number difference ( $K$ being the total spin projection on the nucleus symmetry axis). Hence, we could find processes that are not affected by the $K$ hindrance and that could efficiently deexcite a $K$ isomer. The thermal neutron inelastic acceleration or super-elastic scattering, where some isomer energy is retrieved by the neutron, could be such a process, since the $K$ number is expected to play no role [7] in the decay of the compound nucleus it goes through. Before this work, this process has been measured only two times for ${ }^{152 \mathrm{~m}} \mathrm{Eu}$ [8] and ${ }^{180 \mathrm{~m}} \mathrm{Hf}[9]$.

${ }^{a}$ Presenting author, e-mail: gilbert.belier@cea.fr
In the present paper we report an accomplished indirect measurement of the cross section associated with this process, and a future direct measurement, on the ${ }^{177 \mathrm{~m}} \mathrm{Lu}$ isomer.

The principle of the indirect measurement is to measure in the same neutron spectra both the radiative [10] capture and the burn-up cross sections [11]. Since only the radiative capture and the inelastic acceleration contribute to the isomer burn-up at thermal energies, the difference between the 2 measured quantities is the cross section for neutron inelastic acceleration. Because the conditions for these two kind of measurements are very different many irradiations were made in several tank type reactors (HFR at ILL, OSIRIS and ORPHEE in Saclay) to be able to extract the inelastic scattering cross section. For each irradiation a precise knowledge of the neutron spectra was needed. For the spectra characterization the Westcott convention [12] was used, hence, two parameters were needed: the water temperature, and the epithermal factor $\mathrm{f}$. This last factor was determined by the $\mathrm{k}_{0}$ [13] method using the two isotopes in natural zirconium.

For these 2 measurements the ${ }^{177} \mathrm{Lu}$ isomer has been obtained by thermal neutron irradiation of a highly enriched $(99.993 \%)^{176} \mathrm{Lu}$ powder produced by the mass separator PARSIFAL [14]. Two ${ }^{176} \mathrm{Lu}$ samples with masses 1.25 and $0.31 \mathrm{mg}$ have been irradiated at the ILL high flux reactor (HFR) in Grenoble (France) for 49 days in the V4 irradiation port, where the neutron flux is about $1.5 \times 10^{15} \mathrm{n} \cdot \mathrm{cm}^{-2} \cdot \mathrm{s}^{-1}$ with $15 \%$ non-thermal neutrons. After irradiation, the samples were cooled to remove the short-lived ${ }^{177} \mathrm{Lu}$ ground state (6.647 days) that decays by $\beta$ emission to ${ }^{177} \mathrm{Hf}$, which is then chemically removed [15]. 


\section{Radiative capture cross section}

The ${ }^{178} \mathrm{Lu}$ nucleus is unstable with a half life of 28.4 minutes and it has a $23.1 \mathrm{~m}$ isomer whose excitation energy is $120 \mathrm{keV}$. In order to measure the ${ }^{178} \mathrm{Lu}$ activation the ${ }^{177 \mathrm{~m}} \mathrm{Lu}$ samples were irradiated for 9 to 26 minutes depending on the reactor, before they were gamma counted. Only $\gamma$-lines from the ${ }^{178} \mathrm{Lu}^{\mathrm{m}}$ were observed in the spectra. This can be explained from spin consideration since the capture level $\mathrm{E}_{\mathrm{x}}=6.98 \mathrm{MeV}$ has spins $\mathbf{J}^{\pi}=\left(11^{-}, 12^{-}\right)$and the isomer one is $\mathrm{J}^{\pi}=9^{-}$, whereas the ground state is a $\mathbf{J}^{\pi}=1^{-}$state. A $\gamma$-ray cascade code [16] has been used to follow this $\gamma$ decay path. A very high probability $99.7 \%$ has been obtained for the $\mathrm{K}^{\pi}=$ $9^{-}$isomer in ${ }^{178} \mathrm{Lu}$ feeding. Moreover, the existence of an unknown long lived isomer which could trap a part of the $\gamma$ decays is very improbable, because of the presence of the $\mathrm{K}^{\pi}=9^{-}$isomer with a very low energy that provides an escape path for any possible high K-state. Any other isomer could decay to a rotational state built on this isomer, with low forbiddeness factors $v=\Delta \mathrm{K}-\lambda$, where $\Delta \mathrm{K}$ and $\lambda$ are the transition K-variation and multipolarity. Therefore, such a state should be short-lived and should have been seen in our spectra. This hypothesis is supported by the negative result of a recent experiment [17] searching for new isomers in this nucleus. Hence the measurement of the ${ }^{178 m}$ Lu activation can be used to obtain the total ${ }^{177 \mathrm{~m}} \mathrm{Lu}$ radiative capture cross section.

The lower part of figure 1 shows a fraction of the timeintegrated $\gamma$-ray spectrum obtained after the ILL/H9 irradiation, with two $\gamma$-lines at $325.60 \mathrm{keV}$ and $327.68 \mathrm{keV}$ assigned to ${ }^{178} \mathrm{Lu}^{\mathrm{m}}$ and ${ }^{177} \mathrm{Lu}^{\mathrm{m}}$ decays respectively. By using the ratio between these two $\gamma$-lines area the detection efficiency is cancelled out because the energies are very close. The same elimination is done between the $213.41 \mathrm{keV}\left({ }^{178} \mathrm{Lu}^{\mathrm{m}}\right)$ and $214.43 \mathrm{keV}\left({ }^{177} \mathrm{Lu}^{\mathrm{m}}\right) \gamma$-lines. Hence these ratios can be used to easily extract the ${ }^{178} \mathrm{Lu}$ cross section with no uncertainty associated with the detection efficiency.

Four measurements were done in 4 different neutron spectra and used to perform a Westcott analysis. Since it showed there is no integral resonance for this process and no resonance in the thermal energy domain, the Maxwellian averaged cross section could be extracted from these data:

$$
\sigma_{\mathrm{n}, \gamma}=369 \pm 23 \text { barns. }
$$

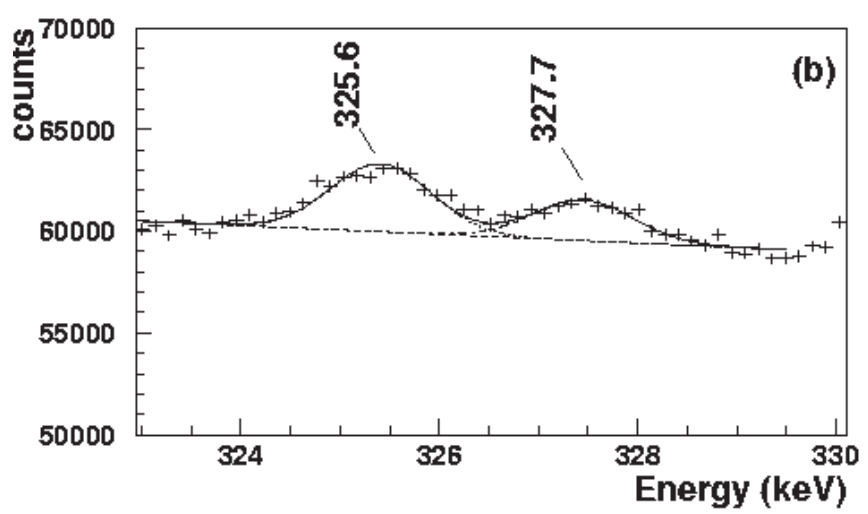

Fig. 1. Partial $\gamma$-ray spectrum obtained after irradiation.

\section{Burn-up cross section}

The burn-up cross section measurements consists in measuring very precisely ${ }^{177} \mathrm{Lu}^{\mathrm{m}}$ activity before and after an irradiation with the same HP-Ge detector set-up. Statistical errors on the number of $\gamma$-ray events as well as solid angle incertitude were controlled to be lower than 5\%. IRMM (Institute for Reference Materials and Measurements, Geel, Belgium) Aluminium monitors loaded at $0.1 \%$ with ${ }^{59} \mathrm{Co}$ [18] were irradiated with each sample in order to measure the total neutron fluence. To perform a precise enough measurement a burn-up of at least $5 \%$ is needed. Hence irradiation durations ranged from 3 days to almost 2 months.

On figure 2 the effect of the isomer burn-up is clearly seen, the dashed line indicating the isomer natural decay. After correction for the natural decay, the burn-up cross section is easily deduced. Here again the Maxwellian averaged cross section could be extracted and the averaged value on two different irradiations is:

$$
\sigma_{\text {burn-up }}=626 \pm 45 \text { barns. }
$$

These direct measurements were completed by indirect measurements of the ground state and the isomer burn-up cross sections, taking benefit of the isomer production irradiation. The cross sections were obtained by measuring these two states populations, knowing the initial ${ }^{176} \mathrm{Lu}$ mass and the half lives and activation cross sections for these 2 states. Cross sections of $880 \pm 75$ and $568 \pm 79$ were respectively obtained for the ground and isomer states.

Finally the inelastic neutron acceleration cross section was deduced from the radiative capture and burn-up cross sections:

$$
\sigma_{\text {inna }}=257 \pm 50 \text { barns. }
$$

All the measured cross sections were obtained for a temperature of $323 \mathrm{~K}$.

\section{Direct measurement}

Since the neutron acceleration cross section is high (the highest measured), a direct measurement can be envisaged.

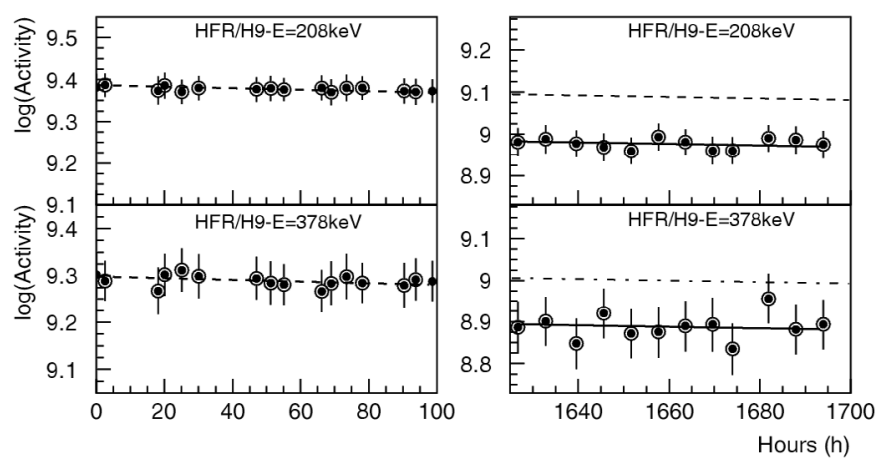

Fig. 2. The logarithm of $228.5 \mathrm{keV}$ and $378.5 \mathrm{keV}{ }^{177} \mathrm{Lu}^{\mathrm{m}} \gamma$-ray activities deduced from $\gamma$-spectroscopy before and after HFR/H9 irradiation at ILL for the $208.4 \mathrm{keV}$ and $378.5 \mathrm{keV}{ }^{177} \mathrm{Lu}^{\mathrm{m}} \gamma$-ray activities. 


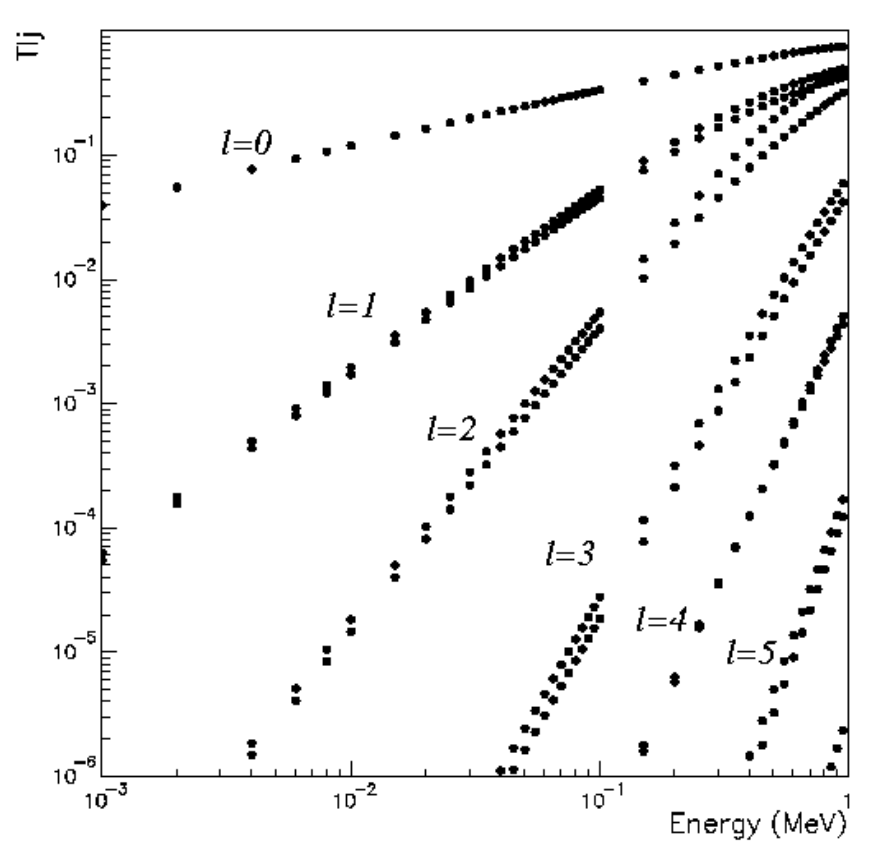

Fig. 3. Neutron transmission coefficients of ${ }^{178} \mathrm{Lu}, \mathrm{T}_{\mathrm{lj}}$, as function of neutron energy for various orbital momentum $l$, at $6.99 \mathrm{MeV}$ obtained by optical model calculations.

Contrary to the first two measurements on ${ }^{152 \mathrm{~m}} \mathrm{Eu}[8]$ and ${ }^{180 \mathrm{~m}} \mathrm{Hf}$ [9] we want to measure the outgoing neutron energies in order to identify the involved transitions in the exit channel.

Using the deformed optical potential developed in Bruyères-le-Châtel [19], calculations were performed to obtain the $\mathrm{S}$-wave neutron strength function $\mathrm{S}_{0}(\mathrm{E})$ and the $\mathrm{T}_{l j}(\mathrm{E})$ neutron transmission coefficients. The $\mathrm{S}$-wave neutron strength function was determined for the ${ }^{177} \mathrm{Lu}$ nucleus in the isomer state $\left(\mathrm{J}^{\pi}=23 / 2^{-}, \mathrm{S}_{0}=1.9886 \times 10^{-4}\right)$ and in the ground state $\left(J^{\pi}=7 / 2^{+}, S_{0}=1.9912 \times 10^{-4}\right)$. In the case of the isomeric target, the transmission coefficients obtained from the optical model for various orbital momentum carried by the neutron are shown as a function of neutron energy in figure 3. For s-wave neutron, the spin of the compound nucleus formed in the reaction ${ }^{177} \mathrm{Lu}^{\mathrm{m}}(\mathrm{n}, \mathrm{n}$ ') could be either $\mathrm{J}^{\pi}=11^{-}$or $\mathrm{J}^{\pi}=12^{-}$. According to the figure 3 , from spin $11^{-}$, the likely neutron transitions correspond to neutron energies of $125.3,334,518.7 \mathrm{keV}$. From spin $12^{-}$, the neutron transmission coefficients are lower than $10^{-5}$.

To perform the direct measurement the ORPHEE reactor has been retained. Here extracted fluxes of $5 \cdot 10^{9} \mathrm{n} \cdot \mathrm{cm}^{-2} \cdot \mathrm{s}^{-1}$ are available. $\mathrm{A}^{177 \mathrm{~m}} \mathrm{Lu}$ target, whose activity is around $9 \mathrm{MBq}$, was obtained by irradiating a $10.8 \mathrm{mg}$ metallic natural $\mathrm{Lu}$ foil at the ILL reactor. In such conditions the expected signal is 225 events/s.

The main difficulty in this experiment is to overcome the low signal to background ratio. Two background sources have to be considered. The first one is due to scattered thermal neutrons. To limit the count rate a dedicated chamber as been built to work under vacuum. The second one is due to neutron capture photons and the gamma activity of the target itself. Here the choice of the detector is of prime importance. An ${ }^{3} \mathrm{He}$ detector has finally been retained. This is a 10 bar proportional

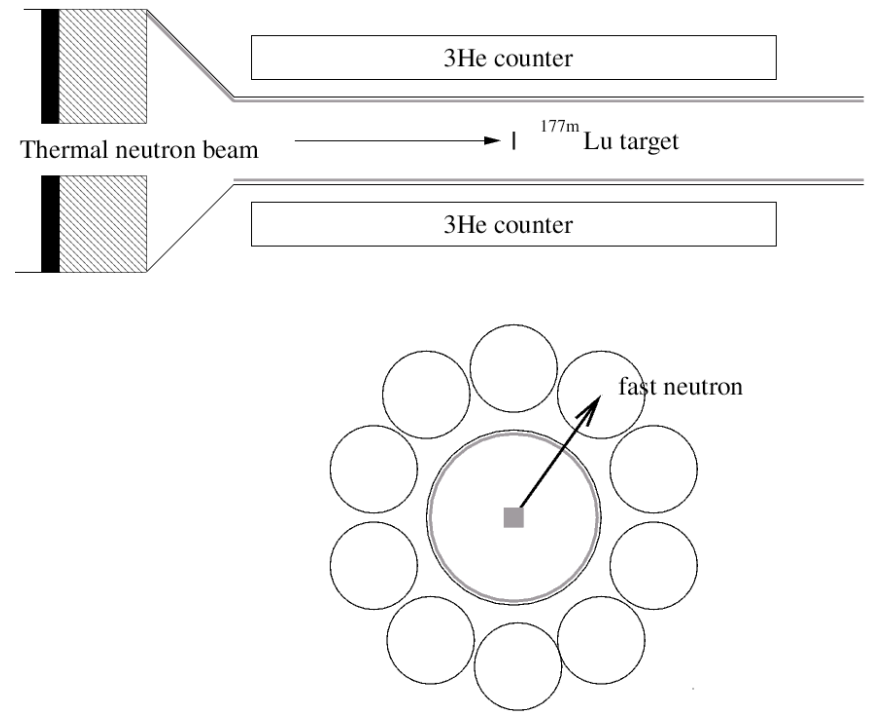

Fig. 4. Schematic view of the direct measurement experiment.

counter from the Saint-Gobain company filled with pure ${ }^{3} \mathrm{He}$. Its outer diameter is 1 inch and its active length is $30 \mathrm{~cm}$. In order to place the detectors as close as possible to the target the neutron beam was collimated to a diameter of $2 \mathrm{~cm}$. The collimator is constituted of a $1 \mathrm{~cm}$ thick boron carbide neutron absorber, followed by a $1 \mathrm{~cm}$ thick $\mathrm{CH}_{2}$ layer and a $2 \mathrm{~cm}$ thick lead gamma attenuator. It is placed at $1.8 \mathrm{~m}$ upstream of the target. A second collimator, made of a $1 \mathrm{~cm} \mathrm{~B}{ }_{4} \mathrm{C}$ and a $5 \mathrm{~cm}$ lead layers, is placed as close as possible to the target in order to eliminate scattered neutrons on the first collimator. Finally 10 detectors can be placed at a distance of around $5 \mathrm{~cm}$ from the target. The inner part of the vacuum chamber as well as the ${ }^{3} \mathrm{He}$ detectors are Cadmium shielded. The total detection solid angle is $24 \%$ and the total absolute efficiency goes from $0.1 \%$ to $0.05 \%$ for neutron from $120 \mathrm{keV}$ to $500 \mathrm{keV}$. Two schematic view of the set-up are depicted on figure 4, with the second collimator, the ${ }^{177 \mathrm{~m}} \mathrm{Lu}$ target and the ${ }^{3} \mathrm{He}$ detectors. In such a configuration the scattered neutron background is only $3 \cdot 10^{-4} \mathrm{n} \cdot \mathrm{cm}^{-2} \cdot \mathrm{s}^{-1}$ while the gamma background was estimated by simulation to be at most $10^{5} \mathrm{ph} \cdot \mathrm{cm}^{-2} \cdot \mathrm{s}^{-1}$. Despite the high photon flux the neutron detection is not affected because the deposited energy is small and associated with a very low efficiency. Moreover the neutron detection is obtained via the exothermic reaction:

$$
\mathrm{n}+{ }^{3} \mathrm{He} \rightarrow{ }^{3} \mathrm{H}+\mathrm{p}
$$

whose $\mathrm{Q}$ value is $764 \mathrm{keV}$.

Tests with a very intense ${ }^{60} \mathrm{Co}$ source showed that the thermal neutron peak located at $764 \mathrm{keV}$ was not affected for photon fluxes up to $10^{7} \mathrm{ph} / \mathrm{s}$ incident on the detector. Finally in the conditions of the measurement the background above the thermal neutron peak is $1.72 \cdot 10^{-2}$ counts/s. It has to be compared to the expected signal around $3.88 \cdot 10^{-2}$ counts/s. The signal to background ratio may appear low but the background corresponds to all energies above the thermal peak, while the signal is expected to be located at precise energies. Hence the described set-up should allow to measure the outgoing neutron energies in inelastic scattering acceleration. 


\section{Conclusion}

A high cross section for thermal neutron inelastic acceleration has been measured. At the same time the radiative capture on the ${ }^{177} \mathrm{Lu}$ has been obtained. We are working now at measuring the outgoing neutron energies. The main goal in this new measurement is to identify the neutron transitions in the exit channel and especially to determine which band is fed. This will enable us to address the question of $\mathrm{K}$ conservation in neutron emission.

\section{References}

1. P. Walker, G. Dracoulis, Nature (London) 399, 35 (1999).

2. E. Lubkiewicz et al., Z. Phys. A 355, 377 (1996).

3. S.A. Karamian et al., Z. Phys. A 356, 23 (1996).

4. I. Bikit et al., Astrophys. J. 522, 419 (1999).

5. G. Rotbard et al., Phys. Rev. C 48, R2148 (1993).

6. S. Deylitz et al., Phys. Rev. C 53, 1266 (1996).

7. Nuclear Structure, edited by A. Bohr, B.M. Mottelson (Benjamin, New York, 1975).
8. I.A. Kondurov, E.M. Korotkikh, Yu. V. Petrov, JETP Lett. 31, 232 (1980).

9. I.A. Kondurov, E.M. Korotkikh, Yu.V. Petrov, G.I. Shuljak, Phys. Lett. B 106, 383 (1981).

10. G. Bélier et al., Phys. Rev. C 73, 014603 (2006).

11. O. Roig et al. Phys. Rev. C 74, 054604 (2006).

12. C.H. Westcott, W.H. Walker, T.K. Alexander, in Proceeding of the 2nd International Conference on Peaceful Use of Atomic Energy, Geneva, Vol. 16, United Nations, New York (1958), p. 70.

13. A. Simonits, F. De Corte, A. De Wispelaere, J. Hoste, J. Radioanal. Nucl. Chem. 113, 187 (1987).

14. L. Maunoury et al., Nucl. Phys. A 701, 286c (2002).

15. O. Roig et al., Nucl. Instrum. Meth. Phys. Res. A 521, 5 (2004).

16. L. Pangault, Ph.D. thesis, Université de Lyon 1 (1999).

17. M.B. Smith et al., Nucl. Phys. A 746, 617c (2004).

18. IRMM, http://www.irmm.jrc.be/html/reference_materials_catalogue/catalogue/cat.pdf.

19. P. Romain et al., in Proceedings of the NEA Specialists Meeting on the Nucleus-Nucleus Optical Model up to 200MeV, Bruyères-le-Châtel, 1996, edited by OECD, Paris (1997), p. 16. http://db.nea.fr/html/science/om200/ 